\title{
Experimental Model In Vivo for Quantitative Assessment of Bone Resorption Inhibition
}

\author{
Ugo E. Pazzaglia, Giovanni Zatti, *Amalia Di Nucci, $\uparrow$ Anna Coci, and Paolo Cherubino \\ Clinica Ortopedica e Traumatologica, II Facoltà di Medicina e Chirurgia dell'Università di Pavia, Ospedale F. Del Ponte, \\ Varese; *Istituto di Farmacologia II dell'Università di Pavia; and †Dipartimento di Patologia Umana ed Ereditaria, \\ Università di Pavia, I.R.C.C.S. Policlinico San Matteo, Pavia, Italy
}

\begin{abstract}
Summary: Quantitative assessment of bone resorption inhibition in vivo is not easily accomplished; methods relying on a count of osteoclasts are questionable, and histomorphometric evaluation of the bone mass presents several technical problems as well. The authors developed a simple method to measure the inhibition of bone resorption by study of the proximal tibial metaphysis of growing rats: the height of the perichondrial bone ring was taken as an index of the balance between osteoblastic and osteoclastic activity because any agent that inhibits osteoclasts (without interference with osteoblasts) produces an increase in the height of this anatomical structure. Since the ring is well demarcated by surrounding tissues, its height can be measured with accuracy and used for quantitative assessment of bone resorption inhibition. This model was tested with salmon calcitonin, and it provides evidence in vivo that this hormone inhibits osteoclastic bone resorption.
\end{abstract}

Drug and hormone inhibitors of osteoclastic activity can be tested in vitro with organ culture techniques $(5,13,18)$ or in cultures of isolated osteoclasts $(2-4,19)$, but application of these results to the in vivo environment, especially with regard to the dose necessary to produce an inhibitory effect, is problematic. Histomorphometric evaluation of the bone mass can be carried out on cortical bone of experimental animals (14); however, due to the relatively slow rate of cortical bone turnover, inhibition of osteoclastic activity is neither quickly nor easily revealed. Morphometry of the metaphyses, where the rate of bone turnover is higher, presents several technical problems that make this method unreliable. The number of osteoclasts has been used as a parameter of bone

Received April 1, 1992; accepted February 22, 1993.

Address correspondence and reprint requests to Dr. U. E. Pazzaglia at Clinica Ortopedica e Traumatologica, II Facoltà di Medicina e Chirurgia dell'Università di Pavia, Ospedale F. Del Ponte, I-21100 Varese, Italy. resorption $(1,7)$; however, when inhibitors are administered, the number of cells no longer correlates with the real extent of bone matrix resorbed.

The study of the proximal tibial metaphysis of growing rats treated with salmon calcitonin provides a simple method to measure the inhibition of bone resorption.

\section{MATERIALS AND METHODS}

Forty Sprague-Dawley rats (Stefano Morini, S. Polo D'Enza, Reggio Emilia, Italy), weighing about $150 \mathrm{~g}$, were used. They were distributed randomly into four groups of 10 animals each. The rats in the first group (A) received $100 \mathrm{U} / \mathrm{kg} / \mathrm{day}$ of salmon calcitonin (Sandoz, Milano, Italy) subcutaneously for 21 days; those in the second group (B) received 50 $\mathrm{U} / \mathrm{kg} /$ day for 21 days; and control group 1 (C1) received the same volume of only the vehicle daily for 21 days. Control group 2 (C2) received the vehicle for 21 days and then were killed 60 days after the last 

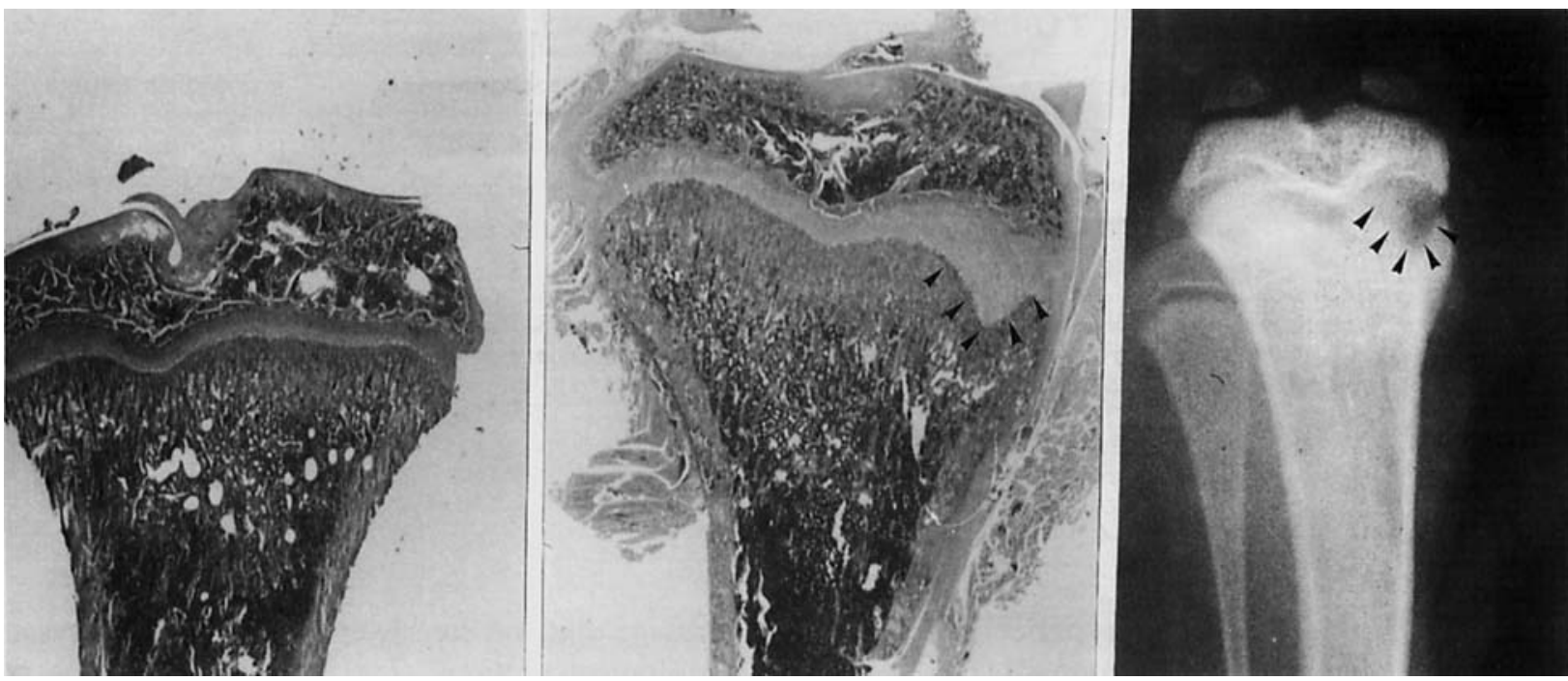

FIG. 1. Low-power view (hematoxylin and eosin, $\times 12.5$ ) of the proximal tibia of control rats (left) and of group $A$ (center). On the medial side, extension of the growth plate inside the metaphysis is present. This is due to persistence of hypertrophic chondrocytes, which have not been invaded by metaphyseal vessels. The intercolumnar septa in this area are not calcified, as can be seen in the corresponding radiograph. The metaphysis has a cylindrical shape, which is a consequence of metaphyseal modeling arrest.

dose. The mean body weight of the $\mathrm{C} 2$ rats at the time of death was $230 \mathrm{~g}$. No significant differences in mean body weight were observed among groups A, $\mathrm{B}$, and $\mathrm{C} 1$ at the end of the study. The rats in these groups were killed at 21 days. The left tibia of each animal in each group was carefully dissected from soft tissues without damage to the periosteum and perichondrium.

The specimens were fixed in neutral formalin $(10 \%)$ and decalcified in EDTA for 2 weeks. The proximal tibia was cut manually with a blade in the median coronal plane. The posterior half of the specimen then was embedded in paraffin, and the plane cut surface was used as a reference for section with a rotatory microtome. Specimens about $10 \mu \mathrm{m}$ thick were stained with hematoxylin and eosin and mounted in routine fashion.

Assessment of the height of the perichondrial ring on the medial and lateral sides was performed on the first section with a ocular grid $(\times 100)$. Its measure was expressed as the mean of the medial and lateral sides. The top end of the ring was identified as the point, just inside the perichondrial groove of Ranvier, where cells crowd on the outer aspect of the ring and a very thin, bony lamina becomes apparent in the context of cartilage. The bottom end was considered to be the net interruption of the ring, resorbed by osteoclasts. A Student $t$ test was applied for comparison among groups.
The numbers of osteoclasts and of nuclei per osteoclast were assessed in each animal as a mean of the count of five sequential sections: the first median coronal section and the following four sections, each $\sim 200 \mu \mathrm{m}$ deeper than the preceding one. The reference area for the count was composed of the epiphysis, the growth plate cartilage, and the metaphysis. The lower limit of the latter was arbitrarily assigned to a line parallel to the growth plate cartilage and at a distance from the proliferative layer equal to twice the thickness of the epiphysis. Only the multinuclear cells on the bone surface were identified as osteoclasts. The count of osteoclasts was expressed as the mean number of cells for five sections of one specimen. The nuclei per osteoclast were expressed as the ratio of the number of nuclei to the number of osteoclasts in the same five sections.

The periosteum and soft tissues were removed from the right tibia. These bones were stored for 7 days in an ethanol-methanol solution $(50 / 50)$ at $37^{\circ} \mathrm{C}$ to extract lipids and water and then dried in an oven at $100^{\circ} \mathrm{C}$ for $48 \mathrm{~h}$. The length of the tibia was assessed with calipers.

\section{RESULTS}

In both groups of rats treated with salmon calcitonin, an arrest of metaphyseal modeling was present, with an inverted concavity of the metaphysis profile 
TABLE 1. Quantitative data on the specimens

\begin{tabular}{ccccc}
\hline Group & Height $(\mathrm{mm})$ & No. of osteoclasts & Nuclei/osteoclasts & Tibial length (mm) \\
\hline A $(\mathrm{n}=10)$ & $2.75 \pm 0.46^{a}$ & $38.40 \pm 18.28^{b}$ & $5.24 \pm 0.37^{a}$ & $34.6 \pm 0.3^{e}$ \\
B $(\mathrm{n}=10)$ & $2.35 \pm 0.38^{a}$ & $32.80 \pm 10.37^{c}$ & $4.98 \pm 0.39^{d}$ & $34.8 \pm 0.7^{e}$ \\
C $1(\mathrm{n}=10)$ & $1.37 \pm 0.24^{e}$ & $13.50 \pm 5.58$ & $4.25 \pm 0.67$ & $35.2 \pm 1.2$ \\
C2 $(\mathrm{n}=10)$ & $1.35 \pm 0.36$ & $13.12 \pm 6.11$ & $4.18 \pm 0.43$ & - \\
\hline
\end{tabular}

The measure is expressed as the mean of the medial and lateral sides of each metaphysis. Rats in group A were treated with $100 \mathrm{U} / \mathrm{kg} / \mathrm{day}$ of salmon calcitonin, and rats in group B were treated with $50 \mathrm{U} . \mathrm{C} 1$ and $\mathrm{C} 2$ are control groups. Groups A and B were compared with $\mathrm{C1}$, and group $\mathrm{C} 2$ was compared with $\mathrm{C} 1$ with use of a Student $t$ test.

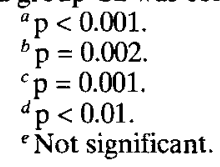

(failure of conization) (Fig. 1). The perichondrial ring was thicker and higher than in group C1 (Fig. 2), and the difference was statistically significant. There was no significant difference in the height of the perichondrial ring between control groups $\mathrm{C} 1$ and $\mathrm{C} 2$ (Table 1). Other features included a persistence of hypertrophic chondrocytes in the outer part of the growth plate, producing a marginal area of thickened cartilage that was clearly evident on both $\mathrm{x}$-rays and histological sections (Fig. 1). The thickening was more evident on the medial aspect of the tibial metaphysis but was present to a lesser degree on the lateral side as well. The central parts of the growth plate and metaphysis, however, were normal. It already has been documented that such changes in the growth plate are due to arrest of vascular invasion

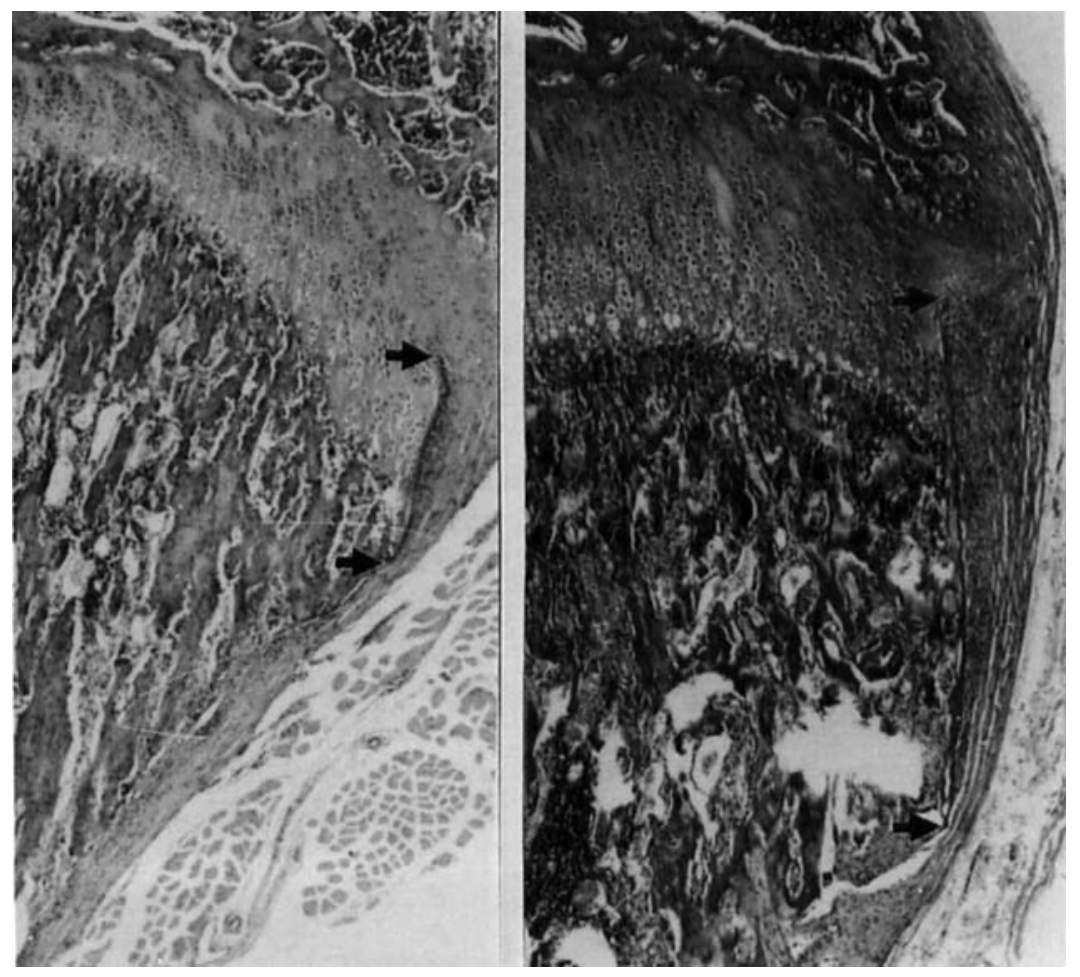

FIG. 2. Histology of the growth plate and metaphysis of control (left) and group A rats (right) (hematoxylin and eosin, $\times 30$ ). The arrows indicate the top and the bottom end of the perichondrial ring; in treated animals, the metaphysis has a cylindrical shape (arrest of metaphyseal modeling), and the ring extends for the entire height of the unmodeled metaphysis. 

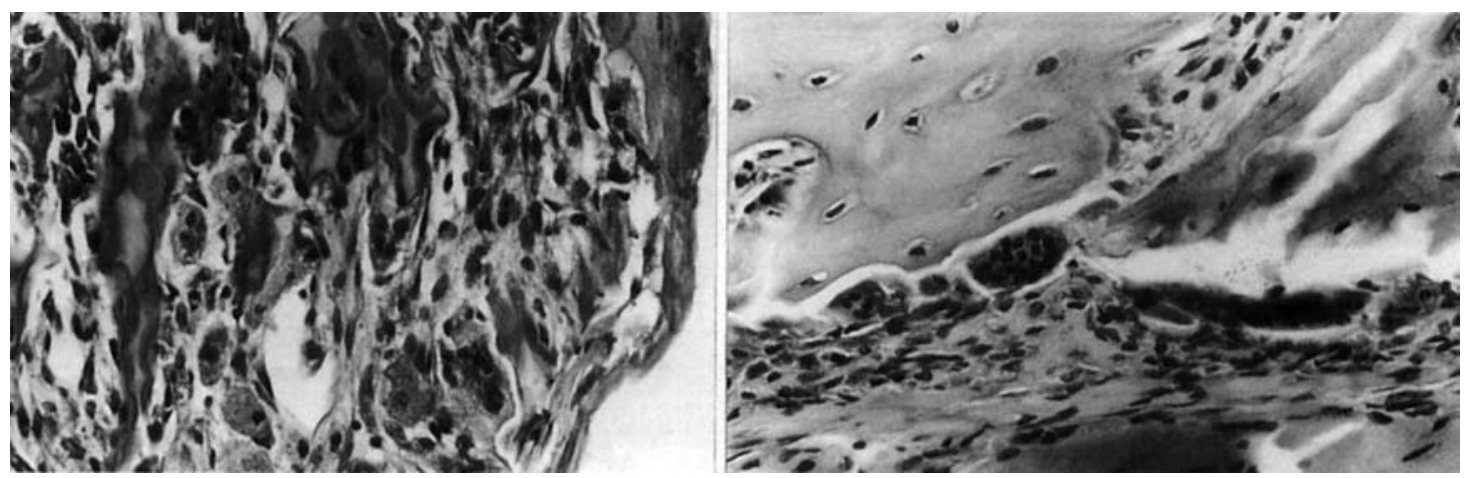

FIG. 3. Comparison of osteoclasts in control (left) and group A rats (right) (hematoxylin and eosin, $\times 400$ ). More nuclei are present in the treated group; the nuclei have a dense chromatin and appear pyknotic.

from the metaphysis (15) and that in this model they are associated with failure of mineralization of intercolumnar septa (10).

Many osteoclasts were present at the distal end of the perichondrial ring and on the outer aspect of the metaphysis (Fig. 3). Their number, as well as the nuclei/cell ratio, was significantly increased in groups $A$ and $B$ (Table 1). The nuclei had a dense chromatin and appeared pyknotic. There were no significant differences in the tibial mean length between groups $\mathrm{A}$ and $\mathrm{B}$ and group $\mathrm{C} 1$ (Table 1).

\section{DISCUSSION}

There is a differentiated structure called the perichondrial ossification groove of Ranvier $(8,12,17)$ at the periphery of the growth plate of developing tubular bones. It consists of an indentation or notch where relatively undifferentiated mesenchymal cells are the progenitors for the osteoblasts forming a thin lamina of bone that encircles the lower portion of the growth plate and is known as the perichondrial ring of Lacroix $(11,16)$. Because of the modeling of the metaphysis (funnelization) in the proximal tibial metaphysis of rodents, as well as in that of other mammals and humans, the tube of bone is not continuous with the periosteal cortical bone of the diaphysis but is instead a separate, distinct ring of bone encircling the lower part of the growth plate and the adjacent portion of the metaphysis.

No osteoclasts or evidence of bone resorption are evident along the outer surface of the ring, whereas osteoclasts are concentrated at the distal end and along the inner surface; therefore, the ring grows upward and outward during funnelization as a result of the synthesis of osteoblasts in the proximal and outer portions (ossification groove). Its height is a function of the rate of osteoblastic activity at its proximal end and of osteoclastic resorption at the distal end. Under healthy conditions, during growth the growth plate and height of the perichondrial ring remain constant. The observation of increased height can be explained only in terms of imbalance of the apposition and resorption rates: either apposition is enhanced or resorption is inhibited. Since there was no significant difference in the total tibial length between treated animals and controls, it can be assumed that the apposition rate was unaffected by calcitonin treatment and that the increased height was due to inhibition of osteoclastic resorption.

A generalized effect on the growth plate was never observed, although it was a constant finding on the marginal parts of the plate. Since the distribution of osteoclasts in the metaphysis is not homogeneous and they are denser at the periphery, where metaphyseal modeling takes place, if calcitonin inhibits osteoclastic activity then its effects should become apparent at sites where the density of osteoclasts is greater and their metabolic activity is faster.

Both ends of the perichondrial ring are well demarcated by surrounding tissues, its height can be measured with a satisfactory degree of accuracy, and, if the apposition rate is not affected by the treatment, the height of the ring allows a quantitative evaluation of inhibition of bone resorption. This model was tested with a well known in vitro inhibitor of osteoclastic resorption, calcitonin, and it allowed a histometric assessment of bone resorption inhibition, which correlates with the dose of calcitonin.

Paradoxically, the number of multinuclear osteoclasts was significantly increased, in contrast with the cylindrical shape of the metaphysis and the enlarged perichondrial ring, which indicated an arrest of bone modeling. Stimulation of osteoclast formation may be 
due to secondary hyperparathyroidism induced by the transient hypocalcemic effect that follows administration of high doses of calcitonin (6). These cells are blocked, however, by the action of the same hormone on the resorbing activity. Therefore, the number of osteoclasts cannot be used as an index of their activity-at least not when an inhibitor of bone resorption is administered. Other features, such as the global shape of the cells and crowding and pyknosis of the nuclei, provide further evidence that these cells are no longer active (9); consequently, no quantitative evaluation of bone resorption may rely on them.

A possible source of error of the assay based on measurement of the perichondrial ring may arise because of the influence of inhibitors not only on osteoclastic resorption but also on the rate of osteoblast synthesis, since in normal bone turnover these processes are coupled. However, an inhibition of osteoblast apposition in growing animals, such as those used in this model, would have resulted in a decrease in the total bone length. An effect on bone length has been observed with other inhibitors of bone resorption, such as EHDP (etidronate) and dichloromethylene diphosphonate, when given to rats at doses of 10 and $30 \mathrm{mg} / \mathrm{P} / \mathrm{kg} /$ day (15). This effect was not observed in the present study; however, it is worthwhile to emphasize that comparison with the tibial length of the control group is a necessary step when the assay is performed.

\section{REFERENCES}

1. Baron R, Vignery A: Behavior of osteoclasts during a rapid change in their number induced by high doses of parathyroid hormone or calcitonin in intact rats. Metab Bone Dis Rel Res 2:339-346, 1981

2. Chambers TJ, Revell PA, Fuller K, Athanasou NA: Resorption of bone by isolated rabbit osteoclasts. J Cell Sci 66:383399,1984

3. Chambers TJ, McSheehy PM, Thomson BM, Fuller K: The effect of calcium-regulating hormones and prostaglandins on bone resorption by osteoclasts disaggregated from neonatal rabbit bones. Endocrinology 116:234-239, 1985
4. Chambers TJ, Fuller K, McSheehy PM, Pringle JA: The effects of calcium regulating hormones on bone resorption by isolated human ostoclastoma cells. I Pathol 145:297-305, 1985

5. Friedman J, Raisz LG: Thyrocalcitonin: inhibitor of bone resorption in tissue culture. Science 150:1465-1467, 1965

6. Glajchen N, Thomas S, Jowell P, Epstein S, Ismail F, Fallon M: The effect of high-dose salmon calcitonin on bone metabolism in the normal rat. Calcif Tissue Int 46:28-32, 1990

7. Hedlund T, Hulth A, Johnell O: Early effects of parathormone and calcitonin on the number of osteoclasts and on serum-calcium in rats. Acta Orthop Scand 54:802-804, 1983

8. Lacroix P: The Organization of Bones. London, Churchill, 1951

9. Miller SC, Jee WS, Kimmel DB, Woodbury L: Ethane-1hydroxy-1,1-diphosphonate (EHDP) effects on incorporation and accumulation of osteoclast nuclei. Calcif Tissue Res 22:243-252, 1977

10. Pazzaglia UE, Zatti G, Di Nucci A, Coci A: Inhibitory effect of salmon calcitonin on bone resorption: morphological study of the tibial growth plate in rats. Calcif Tissue Int 52:125-129, 1993

11. Pratt CW: The significance of the "perichondrial zone" in a developing long bone of the rat. J Anat 93:110-122, 1959

12. Ranvier LA: Traité technique d'histologie, 2nd ed. Paris, Sary, 1889

13. Reynolds JJ, Dingle JT: A sensitive in vitro method for studying the induction and inhibition of bone resorption. Calcif Tissue Res 4:339-349, 1970

14. Russell RG, Kislig AM, Casey PA, Fleisch H, Thornton J, Schenk R, Williams DA: Effect of diphosphonates and calcitonin on the chemistry and quantitative histology of rat bones. Calcif Tissue Res 11:179-195, 1973

15. Schenk R, Merz WA, Muhlbauer R, Russell RG, Fleisch H: Effect of ethane-1-hydroxy-1,1-diphosphonate (EHDP) and dichloromethylene diphosphonate $\left(\mathrm{Cl}_{2} \mathrm{MDP}\right)$ on the calcification and resorption of cartilage and bone in the tibial epiphysis and metaphysis of rats. Calcif Tissue Res 11:196-214, 1973

16. Shapiro F, Holtrop ME, Glimcher MJ: Organization and cellular biology of the perichondrial ossification groove of Ranvier: a morphological study in rabbits.J Bone Joint Surg [Am] 59:703-723, 1977

17. Speer DP: Collagenous architecture of the growth plate and perichondrial ossification groove. J Bone Joint Surg [Am] 64:399-407, 1982

18. Stewart PJ, Stern PH: Vertebral bone resorption in vitro: effects of parathyroid hormone, calcitonin, 1,25 dihydroxyvitamin D3, epidermal growth factor, prostaglandin E2 and estrogen. Calcif Tissue Int 40:21-26, 1987

19. Yamamoto I, Kitamura N, Aoki J, Shigeno C, Hino M, Asonuma K, Torizuka K, Fuji N, Otaka A, Yajima H: Human calcitonin gene-related peptide possesses weak inhibitory potency of bone resorption in vitro. Calcif Tissue Int 38:339341,1986 